\title{
Proceeding
}

Supplementary Issue: Summer Conferences of Sports Science. Costa Blanca Sports Science Events, 25-26 September 2020. Alicante, Spain.

\section{Learning strategy for the formation of tactical thinking in wrestling athletes, category 11-12 years}

\author{
NAHUN DAVID MARTINEZ-SARAVIA ${ }^{1}$, AMINADAN LAÍNEZ-BONILLA¹, JOSE ELIAKIN LAÍNEZ- \\ BONILLA ${ }^{1}$, LIDA DE LA CARIDAD SÁNCHEZ-RAMIREZ² \\ ${ }^{1}$ National Autonomous University of Honduras, Tegucigalpa, Honduras \\ 2University of Oriente, Santiago de Cuba, Cuba
}

\begin{abstract}
Sports activities are essentially complex and require great muscular and mental tensions, therefore, they must be performed by the athlete in a conscious way and this is possible due to the participation of psychological processes, especially thought, which is linked to all sports performance. However, it is currently the least treated from a didactic-methodological approach in wrestling sport. The research was carried out with the purpose of applying a learning strategy for the formation of tactical thinking, which allows the satisfactory execution of tactical actions in situations during the combat of wrestling athletes in the 11-12 year category. The sample was made up of 10 athletes, 15 sports educators and three managers (methodologists at the provincial and national level). Cronbach's Alpha (Internal Consistency Index) was used, determining the reliability of the applied instruments. The research consists of three phases for its development: the first for the determination of the current state of the formation of tactical thinking, from the dimensions and indicators expressed in an individual worksheet; in the second the learning strategy is applied; and in the third, the applicability of the strategy was corroborated through statistical techniques in the comparison before and after its use.
\end{abstract}

Keywords: Didactic; Methodological; Tactics; Learning.

\section{Cite this article as:}

Martínez-Saravia, N.D., Laínez-Bonilla, A., Laínez-Bonilla, J.E., \& Sánchez-Ramírez, L.C. (2020). Learning strategy for the formation of tactical thinking in wrestling athletes, category 11-12 years. Journal of Human Sport and Exercise, 15(4proc), S1456-S1468. doi:https://doi.org/10.14198/ihse.2020.15.Proc4.42

Corresponding author. National Autonomous University of Honduras, 11101 Tegucigalpa, Honduras. https://orcid.org/0000$\underline{0003-1063-9580}$

E-mail: nahum.martinez@unah.edu.hn

Abstract submitted to: Spring Conferences of Sports Science. Costa Blanca Sports Science Events, 19-20 June 2020. Alicante, Spain.

JOURNAL OF HUMAN SPORT \& EXERCISE ISSN 1988-5202

(c) Faculty of Education. University of Alicante

doi:10.14198/jhse.2020.15.Proc4.42

S1456 | 2020| Proc4 | VOLUME 15

(C) 2020 University of Alicante 


\section{INTRODUCTION}

Currently, national and international results in combat sports can show that in the theoretical and methodological order there is a high level of solution for the demands of this sphere, while sports training seeks the development and strengthening of the national reserve (population of children and young people) with talent for sports and are in the training phase for high competition, through adequate preparation at the different levels where it takes place.

However, the tactical training of the aforementioned population is not seen from the beginning in the sport of fighting, an aspect that must be taken into consideration. Although the sciences applied to sport have not addressed in detail the systematicity and depth of said training, the general didactics and didactics of physical education and sport considered valid, in the same way, do not exhaust the particularities of the cognitive process of the thought in its relation to tactical training.

Tactical training must start from primary school and continue without interruption, always adapting to the age of the students. This responds to the pedagogical demands of a reinforcement of mental education and an increase in the quality of primary education (Mahlo, F., 1983).

From the previous comment, fighting sport demands a high development of tactical thinking, not only because of the wide diversity of technical and tactical actions to be solved by the fighting athlete in perceiving, analysing and performing, but because these take place in limited conditions of time and space and must be able to perform analysis and synthesis operations in changing activity situations, since the speed of finding the appropriate solution to a given situation, decision-making and effectiveness depends on the quality of tactical thinking (Gómez C., 2001).

Esquibel W.D. (2003), expresses thought as a cognitive psychic process, which allows man to solve problems, through the operations of thought when faced with each situation, this accounts for the typical features of thought (evidence, metaphorical, speed and intensity and situational).

Related to tactics, studies have been developed on the teaching and learning of sports tactics; for example, learning tactical aspects. Among the studies on the problem of tactical thinking, are: Gómez C. P. (2001), Morales C.Y. (2010), who reaffirm the importance of its treatment. Despite this, in fighting sport it continues to be one of the central problems.

Although the book on technique and tactics of sports fighting by González CS (2013) stands out, the study is directed towards high performance, however, even the authors cited from their theoretical positions, do not condition the orientation of how to achieve the formation of the Tactical thinking during the period of teachinglearning and competitions, in the particularity of school ages.

Studies on tactical thinking carried out by: Puni, A.Z. (1969), Riera, J. (1985), Zatsiosrki, V.M. (1989), Dzhamgarov, T.T. \& Puni A.T.S. (1990); Morales Á.A. \& Col. (2010); Latyshev, S., Korobeynikov, G., \& Korobeinikova, L. (2014). These authors, although their contributions are valuable, do not notice the interrelated behaviour of the fundamental components of the formation of tactical thinking from school ages, but towards high competence from technical-tactical preparation, in addition there is no evidence of a projection of the transformations to be achieved in the short, medium and long term. 
Consequently, there are limitations in the process of building meaningful knowledge, which allows wrestling athletes to advance towards a representation of combat problems, attend to the characteristics of the problem, in disposing and using different actions to solve it. The new scoring system in sport is considered by expanding technical superiority to ten points difference, which allows developing tactical ability and raising tactical thinking.

The research focuses on the formation of tactical thinking, as it constitutes one of the determining aspects for success in the sport of wrestling; where variable efforts predominate with changing tactical situations, therefore it is necessary to study procedures, strategies from learning, which as an educational process becomes a link, for the aforementioned training, and prepares the conditions for the stage of general sports familiarization, until the specialization.

Therefore, the purpose of this study was to apply a learning strategy for the formation of tactical thinking, which allows the satisfactory execution of tactical actions in situations during combat of wrestling athletes, category 11-12 years.

\section{MATERIAL AND METHODS}

To carry out the research, 17 wrestling athletes aged $11-12$ years were selected as the population. 10 were randomly chosen, representing $58 \%$, the sports experience is approximately 2 years.

Also, part of the selection shows 15 wrestling sports pedagogues as specialists, all have degrees in Physical Culture, have 5 or more years of experience in sport and three managers. As users 48 participants in the workshop.

The methodology used in the research is materialized in different stages through which the research passes:

\section{First phase}

Diagnosis of the current state of the formation of tactical thinking in wrestling athletes.

To carry out the documentary study, the objectives were determined, the sample of documents to be studied was established and the analysis indicators were elaborated, to record and evaluate the information obtained. The objective is to assess the treatment given to tactical training and specifically to the training of tactical thinking.

Statistical methods: descriptive (mean, standard deviation, and the coefficient of variation) to process and interpret the results, from the application of the instrument allowed to determine the level of tactical thinking. The following techniques were used: survey and interview directed at sports educators, athletes and managers, as well as observation.

\section{Second phase}

Determination of the dimensions and indicators (Sánchez Ramírez, LC, Loforte Marrón, I. Quintero Cuervo, ME, 2015) for the diagnosis, in the selected sample, the precision of the learning strategy, its functions and the relationships that settle down.

To determine the dimensions and indicators, it was taken into account:

- Information obtained from the detailed study of the specialized bibliography. 
- Theoretical and methodological aspects on the formation of tactical thinking.

- Consult specialists to assess the usefulness of the indicators.

- Evaluation of criteria and suggestions issued by specialists.

The diagnosis followed the following logic:

- Selection of instruments, techniques and methods that allow obtaining relevant information on the status of the process under study.

- Development or adaptation of instruments, techniques and methods in correspondence with the level of knowledge of sports educators and athletes.

- Initial and final diagnosis.

- Processing of the information obtained.

- Integration and interpretation of the results obtained.

- Characterization of the state of the training of tactical thinking of athletes from the selected sample.

Individual Retum

INDICATORS TO MEASURE TACTICAL THOUGHT SPORT WRESTLING

\begin{tabular}{|l|l|l|}
\hline Athlete & Date: & Rival: \\
\hline
\end{tabular}

1. INTERPRETATION OF THE INFORMATION

\begin{tabular}{|c|c|c|}
\hline & Instantly perceive the distance & He manages to perceive distance and action \\
\hline \multicolumn{3}{|l|}{ 1st Half } \\
\hline \multirow{2}{*}{\multicolumn{3}{|c|}{ F FORECASTING OF THE CON }} \\
\hline & & \\
\hline & $\begin{array}{l}\text { Prevents the adversary's intentions } \\
\text { while hiding his true plan }\end{array}$ & $\begin{array}{l}\text { Choose the action properly to seize the moment, } \\
\text { determine the essence of what you perceive }\end{array}$ \\
\hline 1st Half & & \\
\hline 2nd Half & & \\
\hline
\end{tabular}

3. USE OF CONVERGENT MENTAL OPERATIONS logical) and DIVERGENT (creative)

\begin{tabular}{|c|c|c|}
\hline & $\begin{array}{l}\text { It keeps a large part of the actions } \\
\text { under control }\end{array}$ & $\begin{array}{l}\text { It varies its action before } \\
\text { a situation that does not satisfy the achievement } \\
\text { of objectives }\end{array}$ \\
\hline \multicolumn{3}{|l|}{ 1st Half } \\
\hline \multicolumn{3}{|l|}{ 2nd Half } \\
\hline \multicolumn{3}{|c|}{ 4. RAPID AND ACCURATE DECISION MAKING } \\
\hline & Selection of the action in short step & $\begin{array}{l}\text { Keep defensive actions to evaluate situations } \\
\text { that arise }\end{array}$ \\
\hline \multicolumn{3}{|l|}{ 1st Half } \\
\hline 2nd Half & & \\
\hline
\end{tabular}

Figure 1. Individual worksheet to diagnose the state of tactical thinking. Source: Sánchez R.L. (2015) Master's Thesis. 


\section{Third phase}

Evaluation of the applicability of the learning strategy.

Statistical analysis was achieved using the statistical version of the IBM SPSS Statistics 19.0 program. An individual worksheet was used (see Figure 1). A first version was made and it was submitted to the consideration of specialists, then an activity was carried out to prepare the collaborators who would intervene in the observation, later a pilot study was carried out on a small sample, making the pertinent adjustments for its final conformation. Cronbach's Alpha (Internal Consistency Index) was applied to determine the reliability of the applied instruments.

The selection of instruments, such as techniques and methods for the initial and final diagnosis to determine the level of tactical thinking, were used under favourable conditions, a previous preparation was carried out and they were adapted to the characteristics of the investigated subjects.

\section{RESULTS}

The review of the programs of preparation of the fighting athlete for the work of the sports educator, allowed to detect the treatment of tactical training, it is not systemic and is limited in content, in addition to being the same from the first version, issued from the 1981. Said contents only refer to tactical recommendations. It was observed that they do not contribute new elements or actions for the formation of tactics in a systematic way from school ages.

Regarding the results of the application of the survey, almost all of the teacher pedagogues consider that the bibliography consulted in relation to tactical training in specific tactical thinking is insufficient for their specific work. Several of the respondents show a direct correspondence between tactics and technique, however, the former relates directly to psychology.

$87.5 \%$ manifest limitations in the methodological guidelines that allow a direction towards the tactical training of the fighter, for its adequate and profitable use. The existence of barriers to the development of tactical thinking is confirmed, for example, limited theoretical-methodological preparation, insufficient knowledge of problem methods and low level of communication between sports educators and athletes.

In the results of the sports pedagogue survey, information was obtained on the limitations in determining the essential features of the tactic. There are limitations in conceiving tactical actions during the teaching of sport, they value their treatment only through the technical-tactical binomial; there are insufficiencies in distinguishing the difference between tactical and tactical thinking, since only $35 \%$ manage to express their relationship.

Limitations in the use of the problem method, the repetition and global method predominates. $69 \%$ recognize that environmental conditions are not stationary, because they depend not only on the actions of rivals but on intentions.

They argue that there are no indicators to determine the level of tactical thinking in fighting sport. 95\% recognize the attitude of athletes towards the practice of sport as discipline, the desire to be champions and show interest and preference. 
$94 \%$ value watching videos as a way to stimulate tactical thinking, although it is not the most widely used. They give problem teaching an important role in developing thinking and they consider didactic games less significant.

$87 \%$ indicate that the teacher must be aware of active, logical and independent thinking to stimulate tactical thinking. The empirical and the divergent are minimized.

$87 \%$ consider that, to stimulate tactical thinking, communication must be promoted and achieved, and $12 \%$ refer to the need to impose respect, authority and strict control over athletes.

The survey applied to sports educators and athletes selected as a sample was aimed at knowing the level of training of tactical thinking in the sport of wrestling.

Observation was applied in the development of pedagogical activities, with the aim of checking, if they know how to determine the level of tactical thinking from school ages. 5 activities were observed directly and openly, of them 2 in competition, 3 during training.

Regarding the results of the application of the individual worksheet to determine the level of tactical thinking, the Cronbach's Alpha (internal consistency index) is applied, which was 0.88, therefore, the applied instrument is consistent, it is say possesses reliability.

$56 \%$ achieve the necessary conditions in the area (warm, welcoming atmosphere).

The use of the contents and previous experiences is limited to pose problematic situations to athletes. There is an adequate climate of motivation for athletes, interest in playing sports is high. $34 \%$ of sports pedagogues pose problems for athletes to solve.

$36 \%$ have limitations in applying teaching styles and methods for the formation of tactical thinking on offense and defence. $71 \%$ attend to the individual differences of the athletes. Faced with the approach to problem situations, $69 \%$ of athletes start working immediately, $29.1 \%$ analyse ways to resolve the situation.

The self-control and self-assessment of the activity is limited at the beginning, and at the end, only the sports educator points out the main difficulties per athlete. $89 \%$ apply a motivating evaluation.

Results of the considerations of athletes in the teaching of wrestling.

$100 \%$ refer to technical practices as the most performed. $95 \%$ coincide in proposing the predominance of the explanation and demonstration of the sports pedagogue, to the detriment of the observation of videos for the analysis. He prefers to do what the teacher says and does.

$23 \%$ said that problem situations arise and $54 \%$ think before executing them, the rest execute them mechanically. $89 \%$ prefer the execution of techniques in decreasing the approach of situations. $87 \%$ of the respondents express little performance of theoretical activities, and $95 \%$ refer to the performance of difficult activities during the teaching process.

Learning strategy for the formation of tactical thinking in the sport of wrestling (11-12 years). 
Tactical training through learning strategies for athletes is a necessity, because according to existing theories in this regard, it prepares independently through the execution of tactical actions to appropriate the tactical contents, for the formation of tactical thinking mediated by the orientation of the sports educator.

Although, learning strategies are activities, planned according to the needs, sports areas, content, objectives, to be effective learning; These strategies teach athletes to learn and become true teaching styles. This can happen through the order of the sports pedagogue and when the athletes discover the solutions to the variety of situations, presented in the combat, the learning strategies will give part to the learning behaviour and the particular objectives.

The learning strategy assumes the problem method as a teaching-learning method, constituting itself as a mediator between the theoretical-methodological bases built. The didactic essence of the aforementioned method is to facilitate the simulation of situations during combat, athletes can carry out processes of search, discovery, analysis, interpretation, socialization and generalization, to enable the formation of tactical thinking.

The strategy is specified following the assumptions of the systemic-structural-functional method, due to the scientific-methodological need to sequence its stages and actions in an integrated way and guide the procedure in the process of tactical training. The strategy is subject to multiple social influences, in an interactive dynamic to allow its constant redesign and improvement. It is structured in stages that interact with specific actions, establishing hierarchical and subordinate relationships between them.

Methodological requirements for the application of the learning strategy aimed at the formation of tactical thinking (see Figure 2).

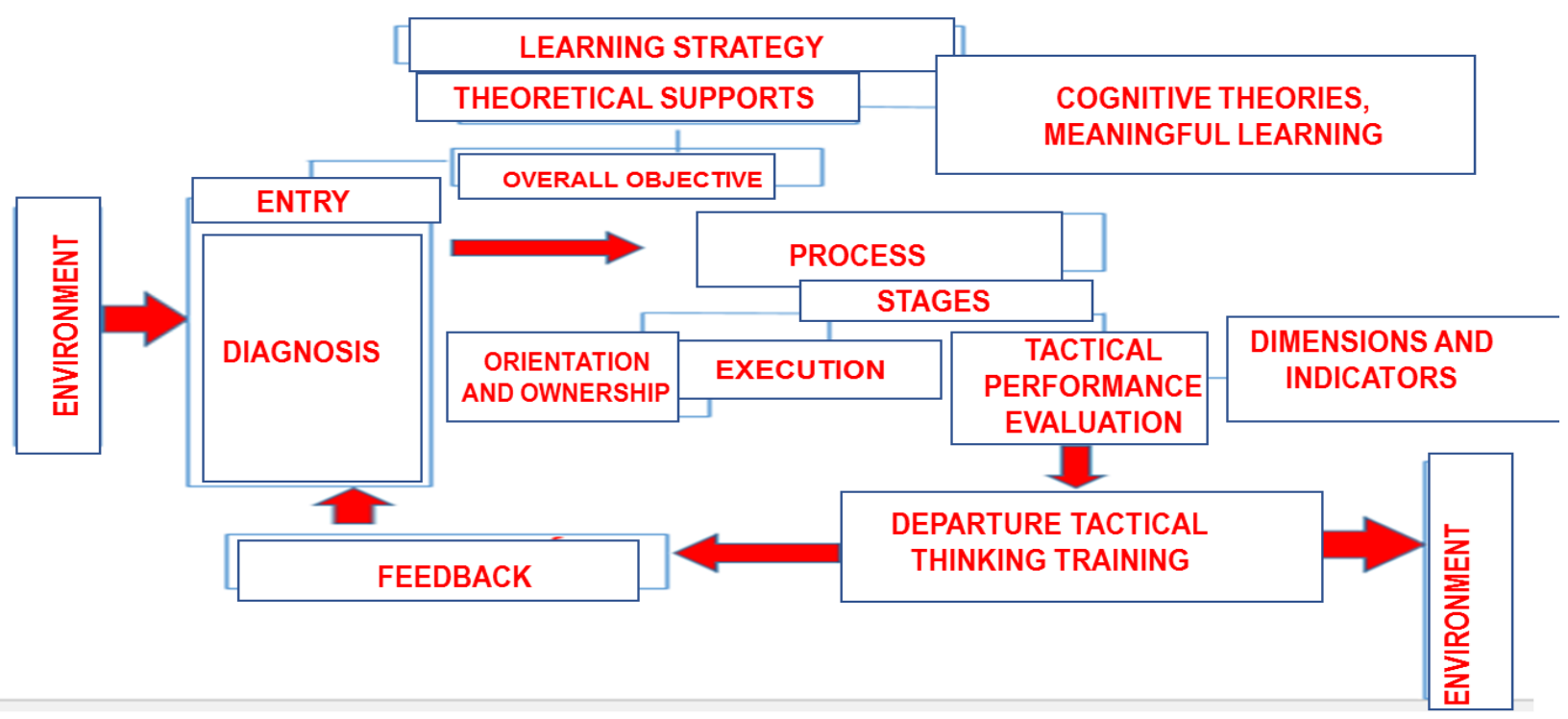

Figure 2. Learning strategy for the formation of tactical thinking of wrestling athletes category 11-12 years. Source: Sánchez R. (2015).

General objective of the learning strategy is expressed in the orientation of the didactic procedure for the implementation of the actions through questions, the execution and the evaluation of their tactical 
performance in the face of the variability of situations in combat, as a concrete expression of the process of formation of tactical thinking.

The strategy comprises four stages, structured and related actions, which enable the formation of tactical thinking in athletes aged 11-12 years in the sport of wrestling. But its level of generality allows it to be adapted for its application to other ages and even to other combat sports where tactics are decisive.

\section{These stages of the strategy}

Stage 1. Diagnosis. Objective: to diagnose the level of training of tactical thinking in wrestling athletes category 11-12 years.

This stage is specified with the application of the instrument developed to determine the level of tactical thinking, containing dimensions and indicators. The sports context where the diagnosis is applied determines the potentialities that must be addressed at this stage, the knowledge of the athletes for the acquisition of tactical knowledge, considering not only the current development area, but the area of immediate development, with levels of help of the sports pedagogue for the tactical training process.

Stage 2. Orientation and appropriation. Objective: to guide towards the appropriation of tactical knowledge that allows the athlete to activate their mental operations and visualize the appropriate strategies according to the diversity of combat situations.

In this stage, the control of their tactical development mechanisms is favoured so that they stimulate their cognitive and practical resources, through what, how, why, with what, when to do, when to apply, consequently, guide the athlete towards a level of conscious appropriation, about how you build your tactical performance and allow an awareness of tactical actions.

To achieve the appropriation of tactical knowledge through questions, the sports pedagogue must stimulate the necessary conditions, in accordance with his previous tactical schemes, to guarantee a process that plans, regulates, mobilizes its patterns and its internal reality, in terms of perception (Tropin, Y., Romanenko, V., \& Ponomaryov, V., 2016) and analysis of the situation (result is knowledge of the situation), mental solution of the problem (result is representation of management), the motor solution of the problem (its result is the practical solution) as an expression of tactical action according to Mahlo F. (1983) in his book "The tactical action of the game" where one of its origins is tactical thinking, although it also There are others such as the breadth of sight, optical-motor calculations, knowledge, reaction speed, motor skills, among others.

This means that, during this appropriation in the wrestling athlete there is a much more conscious dynamization process, not only of their structures and practical schemes to operate satisfactorily during combat situations, based on their tactical performance in the manner more effective, rebuilding it if necessary, but also, its strategies and resources are activated for preparation and leadership in combat.

It is an active process, it is oriented to the activation of strategies and resources for the preparation and conduct of combat, to achieve the perception of distance, perceive distance and action, use convergent (logical) and divergent ( creative), modes of tactical training, explicit models of the activity to be appropriated and the degree of control during the execution of the action, of its relative effectiveness; to channel the intentionality of tactical actions towards a specific objective, not only towards its awareness, but towards its questioning, and to achieve more essential levels of appropriation and internalization from the process of tactical training itself. 
Now, this relationship between diagnosis, orientation and appropriation is expressed in the process of executing tactical action during combat, both in training and in competitions.

Actions:

- Demand explanations and demonstrations.

- Guide and demand the timely confrontation of criteria among athletes in situations in combat. It requires a reflective practice that requires the athlete to specify his goals, analyse what he needs to do to achieve it from the physical, technical, theoretical, and tactical, what he has for it and how he can do it.

- Perception of distance. Determine if the distance to carry out the combat is correct (medium, short and long). Specify the action to take, according to the tactical knowledge learned.

- Perceive distance and action.

- Identify the intentions of the adversary and know how to take advantage of the moment.

- Use of convergent (logical) and divergent (creative) mental operations.

Reflection questions are asked about the actions in the face of the situation and the opposite; correct when the objectives are not met, question the preparation in terms of physical, tactical, psychological and theoretical, individually and the adversary. In addition, pose problematic situations.

Stage 3. Execution. Third stage: execution of the tactical action. Objective: to develop the strategy in practice through actions.

In the execution, it is where the perception, orientation and appropriation of the practice of sport are experienced, the cognitive resources of tactical training are mobilized, their intentionality is projected through the learning strategy.

In this stage, the partial automation and regulation of the operations that is the driving habit are produced, directed to the expected result; Consequently, in sport, to determine if the level of motor habit has been reached, the athlete is asked questions during the execution of the exercise, in this way he must pay attention to the answers; This allows them to develop their cognitive capacities for reflection, criticism and assessment.

Actions:

- Develop a tactical plan for each athlete.

- To confront what has been done with other athletes through the modelling of combat situations and their solution on the mental plane (ideo-motor training) from:

- Videos.

- Situations to demonstrate.

- Orient athletes towards essential aspects of tactical knowledge through questions and tasks that require reflection.

- Guide situations in which the athlete reflects the understanding of tactical knowledge from solving new problems.

In this stage the development of individual qualities of thought is guaranteed: breadth, depth, independence, flexibility, consistency and speed; to achieve an optimal level of consciousness in mental operations and decisions, which are carried out when solving a task, explaining with situations, and asking questions. 
Stage 4. Evaluation of the tactical performance. Objective: evaluate tactical performance.

This refers to the fact that the sports pedagogue conducts the evaluation, checks the level of the athlete's tactical thinking, the results of which make it possible to analyse and assess their own performance.

Evaluative criteria for athletes.

Training level of tactical thinking for solving problems in combat situations during competition and teaching. Achievement patterns for athletes:

- Demonstrate the appropriation of tactical knowledge through heuristic procedures and the execution of tactical actions.

- Demonstrate tactical reasoning ability in the different experiences of applying tactical knowledge to solve problems.

Although the designed strategy complies with the basic properties and requirements set forth in the bibliography related to systems theory, it is not a finished proposal, because dialectically it must be adapted to the logical transformations that appear in the environment for which it was created. Not to mention the new research and experiences to print a higher level of news.

The interpretation of the different frequencies was carried out before the strategy was applied (see Figure 3). 10 athletes were observed, 6 of them were diagnosed with a low level of tactical thinking, finding limitations in perceiving and analysing the moment, distance and action according to the situation, difficulties in preventing the adversary's intentions while hiding their true plan $(78 \%)$, poor recognition to properly choose the action and take advantage of the moment; $89 \%$ have inefficient use of learning strategies to vary the action in the face of a situation that does not satisfy the achievement of the objectives; short-term stock selection limitations; 4 have a medium level, representing $40 \%$. The mean of tactical thinking behaved around the low level.

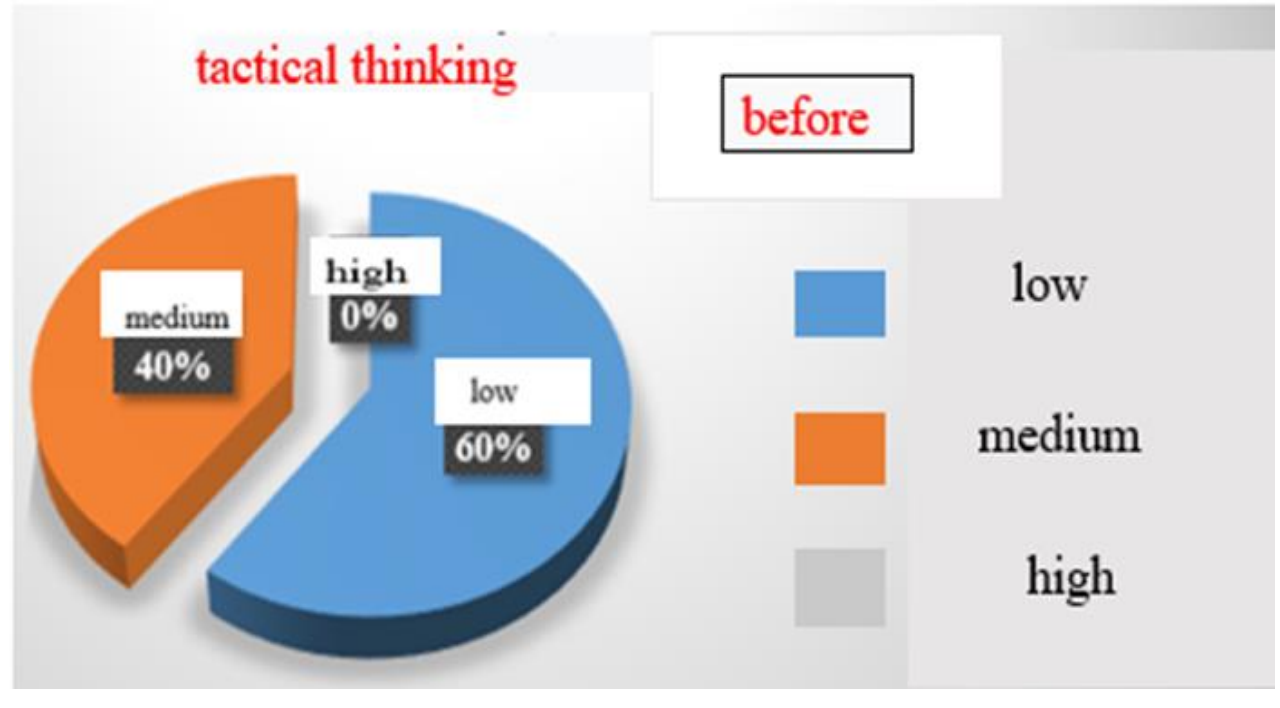

Figure 3. Graph on initial study of the level of tactical thinking. Source: own elaboration based on the observation made. 
From the data obtained, it can be seen that, with a low level of tactical thinking, they can be the causes of inadequacies in the execution of tactical actions during combat.

In the results during the interview with managers, it was agreed that there were limitations in tactical training at ages $11-12$ years. $100 \%$ consider the scarce application of problem methods.

They agree in stating that, during teaching, the study or information about the adversaries is limited. Barriers are expressed in the cognitive domain. 100\% consider the application of problem methods a way to achieve the formation of tactical thinking in athletes, incorporating strategies for self-assessment of their action, and that of the opposite. $100 \%$ pose limitations on sports pedagogues to develop tactical training.

As a summary, the current state of the formation of tactical thinking was diagnosed, detecting shortcomings related to tactical knowledge and the practical application of essential indicators for the work of sports educators.

The observation was made from the application of the proposal when in the teaching process the teacher puts two athletes to fight and arbitrate others, then each one expressed his criteria on the errors and possible variants before the variability of the situations encountered in combat so as not to make the same mistakes again (see Figure 4).

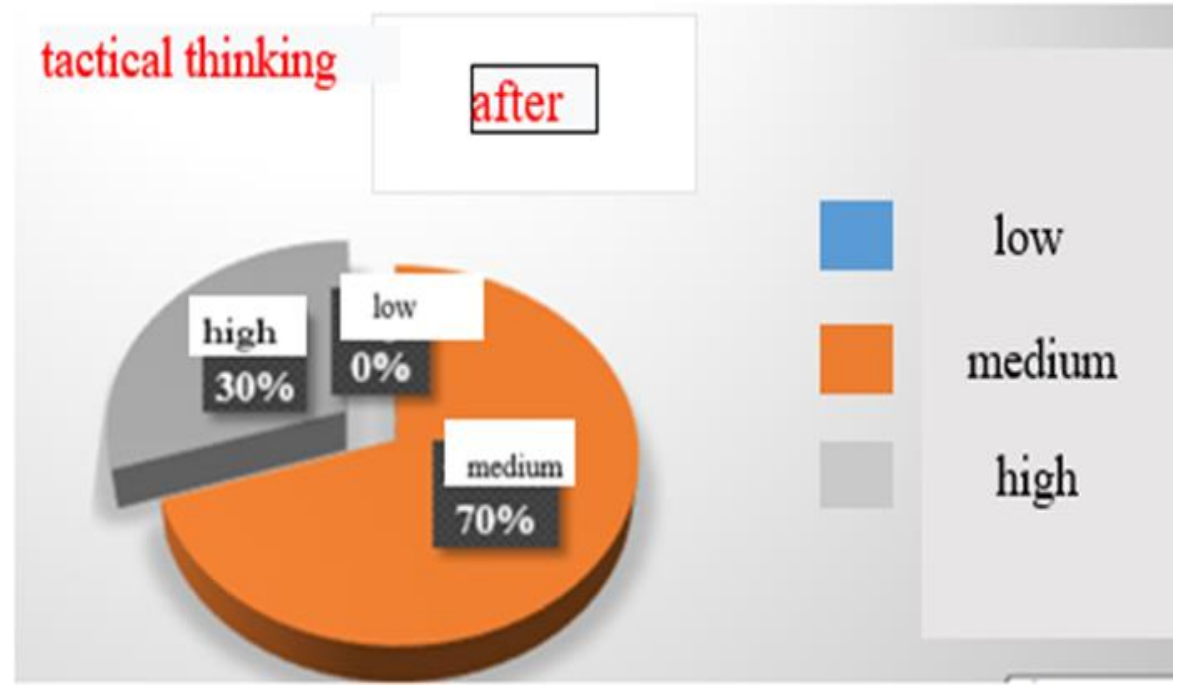

Figure 4. Graph on final study of the level of tactical thinking. Source: own elaboration based on the observation made.

The simple comparison of the percentage of the results from the application of the proposal focuses on the interpretation of the different frequencies. The final study yielded seven athletes with a medium level of tactical thinking. Three with a high level were determined, representing $30 \%$ from the application of the learning strategy; and the satisfactory execution of tactical actions is achieved in situations during combat, say, the highest percentage is between medium and high, and learning strategies may be a proposal to mitigate the shortcomings presented before the execution of tactical actions during combat. Combat. The mean of tactical thinking behaved around the mean level. 


\section{DISCUSSION}

Authors such as Latyshev, \& Korobeynikov, 2013; Latyshev, Korobeynikov, \& Korobeinikova, (2014) recognize that the tactical possibilities in the fighter depend mainly on their physical preparation. However, the learning content must be contextualized and functional through questions raised by López Ros (2014), why do I do this? And what is it for me? The reference by Serra Olivares, J., García López, LM, Calderón, A., \& Cuevas Campo, R. (2015) which express the relationship of tactical knowledge with age, experience and the level of expertise. Sánchez R. (2015) refers that, in the formation of tactical thinking, one must take into account levels of difficulty, task orientation, types of aids, and the necessary interrelation between procedural and explanatory knowledge, these being, to be considered of the authors of this study, the fundamental axes of the process in the initial stages and of tactical training.

It is not enough that the wrestling athlete consciously appropriates tactical knowledge, and dominates through words and actions how to interact with him, but rather to be consistent with tactical action, and to be aware of strategies, resources and potentialities to be able to self-regulate their tactical action, this will then allow a true formation of the content that is awarded in the process of formation of tactical thought.

Thus, the formation of tactical thinking in wrestling athletes in the ages of 11-12 years should refer to a process of construction of meaningful knowledge, to allow progress towards a generic and abstract representation of problems, address the characteristics of the problem, have and use different alternatives to solve it, use of creative capacity, anticipate the action according to Mhalo (1983), to be carried out on the contrary, from certain signals or indications and where the taking successful decision-making.

In summary, to characterize tactical thinking is to recognize the tactical content, by reflecting the basic operations of analysis-synthesis, comparison, generalization, concretion and abstraction thinking, since, to react to the changes arising at the precise moment, the fighter you must think and analyse each tactical manoeuvre. It is recognized that the fundamental characteristic of tactical thinking is the anticipation of the situation.

\section{CONCLUSIONS}

The study of tactical thinking as a representation of tactics is necessary because, from the new changes in the rules, the fight increases its rhythm and intensity, causes an increase in the preparation of technical and tactical actions that energize the top, this forces to look for new and varied combinations for greater effectiveness and development of the tactic. However, despite these considerations, it is the least treated from the didactic-methodological point of view.

The determination of the current state of the problem through the diagnosis made, allowed to identify the existing limitations in the formation of tactical thinking during combat situations created in the field of teaching and skills, these deficiencies can be treated and solved through of a learning strategy.

The results obtained from the application of the strategy provide favourable evidence for the purpose of this study, since it is appreciated that the rigorous and constant attention of the strategy for the formation of tactical thinking by having an adequate transition of tactical knowledge towards its generalization From a didactic approach, it is in a position to raise the indicators for tactical training in the ages of 11 to 12 years. 


\section{REFERENCES}

Dzhamgarov, T. T. and Puní A. T. S. (1990). Psychology of Physical Education and Sports. City of Havana, Technical Scientific Editorial.

Esquibel W. D. (2003). Athlete's brain training. psychopedagogical approach. City of Havana, Technical Scientific Editorial.

Gómez C. Pedro. (2010). Tactical preparation in combat sports. [Computation program].

González C. S. et al. (2017) Types of tactics and their use in sports fighting. Readings: Physical Education and Sports, Digital Magazine. Buenos Aires, Year 22, No. 231.

González, C. S. (2013) Technique and Tactics of Sports Fighting. Edit. Sports. Havana. Cuba.

Latyshev, S., Korobeynikov, G., and Korobeinikova, L. (2014). Individualization of training in wrestlers. International Journal of Wrestling Science, 28-32. https://doi.org/10.1080/21615667.2014.954488

Mahlo, F. (1983). Tactical action in the Game. Havana: People and Education.

Morales A. A. et al., (2010). Method for tactical preparation. A contribution to face the challenge of the intelligent, creative and independent athlete [Computer program].

Morales C. Y. (2010). System for the didactic treatment of the technical-tactical preparation of the school taekwondo player.

Puni, A. Z. (1969). Essay of Sports Psychology. Havana, INDER.

Riera, J. (1985). Introduction to the psychology of sport. Barcelona, Editorial Martínez Roca.

Sánchez R. L. (2015). The formation of tactical thinking in wrestling athletes, category 11-12 years. (Master's Thesis). Eastern University. Faculty of Physical Culture "Manuel Fajardo Rivero". Santiago de Cuba, Cuba.

Sánchez Ramírez, L.C., Loforte Marrón, I. Quintero Cuervo, M.E. (2015). Dimensions and indicators to measure tactical thinking in combat sports. Arrancada, Vol. 15 No. 28 (2015) pp. 54-63.

Serra Olivares, J., García López, L. M., Calderón, A., and Cuevas Campo, R. (2015). Relationship of young soccer players' tactical knowledge with age, experience, and skill level. Notebooks of Sports Psychology, 15 (3), 105-112. https://doi.org/10.4321/S1578-84232015000300010

Tropin, Y., Romanenko, V., and Ponomaryov, V. (2016). Model characteristics of sensorymotor reactions and perceptions of specific wrestlers of different styles of confrontation. Slobozhanskyi herald of science and sport, 70-73.

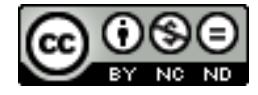

This work is licensed under a Attribution-NonCommercial-NoDerivatives 4.0 International (CC BY-NC-ND 4.0). 